\title{
ENTIERRO SECUNDARIO MÚLTIPLE EN EL CHACO AUSTRAL. SITIO PAVENHAN (VERA, SANTA FE)
}

\author{
MULTIPLE SECONDARY BURIAL IN SOUTHERN CHACO. PAVENHAN SITE \\ (VERA, SANTA FE)
}

\author{
Marcos Plischuk ${ }^{1,2 *}$; Bárbara Desántolo2; Rocío García Mancuso1,2; Carlos Ceruti³; Guillermo \\ Lamenza ${ }^{1,4}$
}

${ }^{1}$ Consejo Nacional de Investigaciones Científicas y Técnicas (CONICET)

${ }^{2}$ Cátedra de Citología, Histología y Embriología “A”. Facultad de Ciencias Médicas. Universidad Nacional de La Plata (UNLP). La Plata. Argentina

${ }^{3}$ Museo de Ciencias Naturales y Antropológicas Profesor Antonio Serrano. Paraná. Entre Ríos. Argentina

${ }^{4}$ División Antropología. Facultad de Ciencias Naturales y Museo. UNLP. La Plata. Argentina

\section{PALABRAS CLAVE: entierro secundario; arqueología de rescate; Chaco austral}

\begin{abstract}
RESUMEN La modificación antrópica del ambiente provocada por la acción agrícola intensiva genera fenómenos erosivos que posibilitan la localización de antiguos espacios de actividad humana. En este trabajo damos a conocer el hallazgo de un sitio arqueológico puesto en evidencia por maquinaria agrícola, situado a $40 \mathrm{~km}$ al oeste de la localidad de Margarita (departamento de Vera, provincia de Santa Fe). El sitio arqueológico fue denominado Pavenhan, y se caracteriza por la presencia de restos óseos humanos con una datación radiocarbónica de $580 \pm 40$ años ${ }^{14} \mathrm{C}$ AP. Una vez realizada la exhumación, los restos fueron acondicionados y ordenados según un criterio anatómico. El material se encontró fragmentado por factores postdepositacionales naturales y antrópicos. Asimismo, se destaca la presencia de marcas de orientación transversal sobre diáfisis media de huesos largos, producidas
\end{abstract}

en su mayoría por roedores. Se procedió a la determinación del número mínimo de individuos $(\mathrm{NMI}=15)$ identificándose adultos $(n=13)$ y subadultos $(n=2)$. Debido al estado de preservación del material, solo se pudo determinar el sexo de ocho individuos, de los cuales cinco se clasificaron como masculinos y tres como femeninos. Las patologías identificadas sobre los restos esqueléticos resultaron ser en su mayoría infecciones inespecíficas, mientras que aquellas asociadas a la cavidad bucal fueron caries y periodontitis. Estos resultados confirman que las sociedades cazadoras recolectoras pescadoras del Chaco austral practicaban enterratorios secundarios, como así también refuerzan la idea de que estos grupos contaban con una incorporación incipiente de hidratos de carbono durante los momentos previos a la conquista española. Rev Arg Antrop Biol 22(2), 2020. doi: 10.24215/18536387e021

\section{KEYWORDS secondary burial; rescue archaeology; southern}

ABSTRACT The anthropic modification of the environment caused by intensive agriculture generates erosive phenomena that make it possible to locate ancient areas of human activity. In this work we present the finding of an archaeological site brought to light by agricultural machinery, located $40 \mathrm{~km}$ west of the town of Margarita (department of Vera, province of Santa Fe). The archaeological site was named Pavenhan, and it is characterized by the presence of human bone remains with a radiocarbon date of $580 \pm 40$ years ${ }^{14} \mathrm{C} \mathrm{AP}$. After the exhumation, the remains were prepared and sorted out according to anatomical criteria. The material was found fragmented by natural and anthropic post-depositional factors. Also, the presence of transverse orientation marks on the middle diaphysis of long bones, produced mostly by rodents, was observed. We proceeded to determine the minimum number of

A inicios del año 2009, a partir de la realización de actividades agrícolas de nivelación mecánica del terreno en la estancia Pavenhan (departamento de Vera, provincia de Santa Fe) (Fig.1), quedaron al descubierto significativas evidencias de presencia de restos óseos humanos. individuals $(\mathrm{MNI}=15)$, identifying adults $(\mathrm{n}=13)$ and subadults $(n=2)$. Due to the state of preservation of the material, sex was determined only in eight individuals, five of which were classified as masculine and three as feminine. Pathologies were identified on the skeletal remains; in their majority, they were nonspecific infections, while those associated with the oral cavity were caries and periodontitis. These results confirm that the hunter-gatherer-fishing societies of southern Chaco practiced secondary burials, and they also reinforce the idea that these groups had begun to include carbohydrates in their diet before the Spanish conquest. Rev Arg Antrop Biol 22(2), 2020. doi: 10.24215/18536387e021

Financiamiento: Universidad Nacional de La Plata (Arqueología y paleoambiente en el Chaco argentino, Proyecto 11/ N846).

*Correspondencia a: Marcos Plischuk. Cátedra de Citología, Histología y Embriología "A". Facultad de Ciencias Médicas. Universidad Nacional de La Plata. Calle 60 y 120. 1900 La Plata.Argentina. E-mail: marcosplischuk@yahoo.com.ar

Recibido 5 Agosto 2019; aceptado 11 Noviembre 2019

doi: $10.24215 / 18536387 \mathrm{e} 021$ 


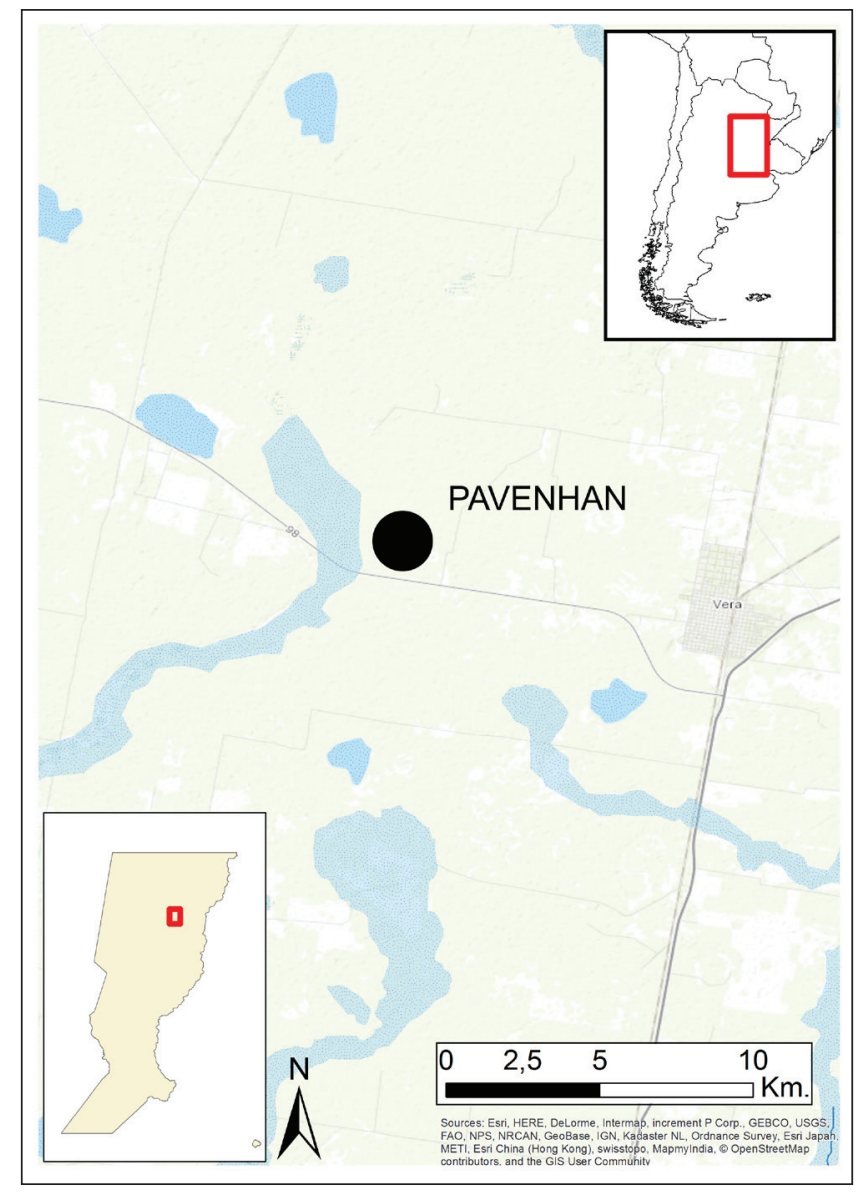

Fig. 1. Localización del sitio arqueológico Pavenhan.

El hallazgo arqueológico fortuito se encontró en vinculación con la Laguna El Palmar, y fue comunicado inmediatamente, tal como es norma en estos casos, a las autoridades municipales competentes. En dicha oportunidad el director del Museo Municipal de Arqueología y Paleontología de la ciudad de Reconquista, Profesor Dante Ruggeroni, fue el encargado de responder y dar inicio a las tareas de rescate y estudio preliminar del material. Se procedió a la exhumación de los restos con una estrategia de recuperación en bloque y por unidades anatómicas individuales, para su posterior traslado y almacenaje en un depósito adecuado para tal fin en las instalaciones del museo. Una vez ingresados los restos como acervo patrimonial de la institución, se solicitó su análisis al equipo de investigaciones antropológicas forenses de la División Antropología del Museo de la Plata (Facultad de Ciencias Naturales y Mu- seo, Universidad Nacional de La Plata). Realizadas las gestiones correspondientes se trasladaron los restos en carácter transitorio a fin de proceder a su estudio. Por lo tanto, en esta oportunidad se presentan los resultados del análisis del material osteológico recuperado, considerando el estado de preservación del material, la descripción demográfica de la muestra y su análisis paleopatológico. Por último se propone una interpretación preliminar del contexto a fin de poner en discusión la práctica inhumatoria representada en el marco de la problemática arqueológica regional.

\section{MATERIAL Y MÉTODOS}

El sitio arqueológico denominado Pavenhan, se encuentra ubicado en la estancia homónima, y el rasgo que lo caracteriza es la alta densidad 
de restos óseos humanos con escaso material arqueológico acompañante. Los elementos óseos presentaban una disposición particular, por un lado cráneos agrupados en un extremo de la concentración y, por otro, las extremidades inferiores, insertados de manera vertical en forma de empalizada (Ruggeroni comunicación personal, 2010). Cabe destacar que los restos se hallaron a poca profundidad, casi superficialmente, motivo por el cual la maquinaria agrícola provocó fracturas en la mayoría de los huesos al descubrir el sitio (Fig. 2).

Una vez en el laboratorio de la División Antropología se procedió a la limpieza y acondicionamiento de los restos, tarea que consistió en la separación de sedimentos adheridos mediante el uso de cepillos de cerdas blandas en seco. El ordenamiento e inventario se realizó siguiendo criterio anatómico, considerando la morfología, morfometría y lateralidad de los huesos, así como sus patrones de articulación (Mays, 1998; Tuller y Hofmeister, 2014; White, Black y Folkens, 2012). Para la cuantificación se realizó el cálculo de abundancia taxonómica en forma de NISP (number of identified specimens per taxón) (Lyman, 1994) y MNI (minimum number of individuals) (White, 1953). Para aproximar la antigüedad de los restos se seleccionaron fragmentos de costilla de escaso valor diagnóstico y se remitieron al Laboratorio de Radiocarbono (CIG, CONICET, UNLP) a fin de realizar una datación radiocarbónica.
Los procesos tafonómicos actuantes sobre los materiales rescatados fueron evaluados a través de la determinación del grado de meteorización siguiendo el criterio de Behrensmeyer (1978); búsqueda de signos de termoalteración y depositaciones químicas, análisis de fracturas y marcas (pre, peri y/o postmortem) e identificación del agente causante (acción antrópica, raíces, carnívoros, roedores, entre otros) (Lloveras, Rissech y Rosado, 2016; White et al., 2012).

Para obtener el perfil demográfico de la muestra de procedió a la caracterización biológica de los individuos. En primer lugar, cada elemento óseo inventariado fue asignado a una categoría de edad (adulto o subadulto) en función de los criterios: tamaño de la pieza, grado de unión epífisis-diafisiaria (Scheuer y Black, 2000), cierre de sutura esfenobasilar (Galera, Ubelaker y Hayek, 1998) y erupción dentaria (Ubelaker, 1999). Dentro de la categoría subadulto, la estimación particularizada de la edad se realizó a partir de la longitud de los huesos largos (Scheuer y Black, 2000) y de la secuencia calcificación - erupción dentaria (Buikstra y Ubelaker, 1994; Moorrees, Fanning y Hunt, 1963). En el caso en que los huesos largos se encontraban fragmentados solo se tomaron mediciones como anchos de los extremos proximal y distal de húmero, radio, fémur y tibia como base para estimar una edad mínima (Cardoso, Vandergugten, y Humphrey, 2017; Hoppa y Gruspier, 1996). Los individuos identificados

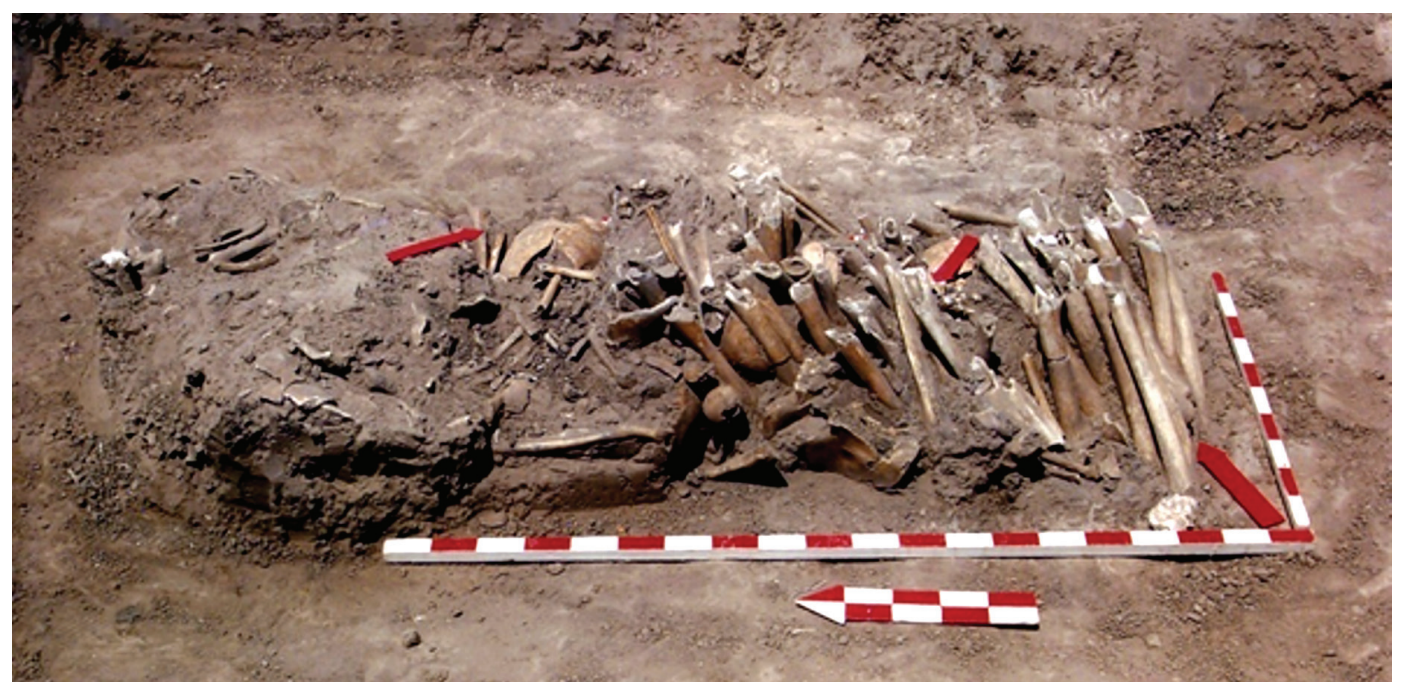

Fig. 2. Disposición de los restos en el espacio inhumatorio. 
como subadultos fueron clasificados en fetales, infantiles (0-3 años), niños (3-12 años) y adolescentes (12-20 años). La determinación del sexo se realizó en forma diferencial según la categoría de edad previamente establecida. En los restos de individuos adultos el indicador de sexo considerado en todos los casos fue el tamaño y forma del proceso mastoideo dada su abundancia en la muestra y el elevado deterioro de los coxales hallados. En los cráneos completos se relevó además la robustez mandibular, los arcos supraorbitarios y la forma de la glabela (Buikstra y Ubelaker, 1994; White et al., 2012).

El análisis paleopatológico fue realizado mediante la observación macroscópica con la ayuda de una lupa 10X. Se describieron los rasgos anómalos para cada hueso y se estableció, en los casos en los que fue posible, su correspondiente hipótesis diagnóstica (Campillo, 2001; Waldron, 2009). El registro de osteoartrosis se llevó a cabo considerando 10 áreas articulares (Waldron, 2009): témporo-mandibular, acromio-clavicular, esterno-clavicular, columna vertebral, gleno-humeral, húmero-radio-ulnar, mano, coxofemoral, fémoro-patelo-tibial y pie, evaluando su severidad mediante la propuesta de Weiss (2006) en grados 0 (ausente), 1 (leve), 2 (moderado) y 3 (severo). Para evaluar las características de las hernias discales observadas se siguieron las consideraciones de Jiménez-Brobeil, Souich y Al Oumaoui (2009) y Üstündağ (2009). Los signos de estrés metabólico relevados fueron lesiones craneales en bóveda y órbitas (hiperostosis porótica y cribra orbitalia) e hipoplasias del esmalte dental (Hillson, 2000; Ortner, 2003; Stuart-Macadam, 1989). El resto de las enfermedades que involucraron a la cavidad bucal se evaluó siguiendo los criterios de Hillson (2000). Se relevaron señales de trauma (Lovell, 1997) e infecciosas específicas e inespecíficas en cráneo y postcráneo (Ortner, 2003; Waldron, 2009).

\section{RESULTADOS}

La antigüedad de los restos fue estimada a partir de la realización de una datación radiocarbónica que dio como resultado $580 \pm 40$ años ${ }^{14} \mathrm{C}$ AP (LP2364, Homo sapiens). La calibración en años calendario se realizó a través del programa CALIB 7.1 con la curva para el hemisferio sur SHCal13 (Hogg et al., 2013). Los rangos de edad calibrada son 1393-1434 cal AD (1 sigma, $\mathrm{p}=1), 1319-1351$ cal AD (2 sigma, $\mathrm{p}=.177)$ y 1385-1446 cal AD (2 sigma, $\mathrm{p}=.823)$ con una edad promedio de 1407 años AD.

El material se encontró muy fragmentado, presentando una meteorización general mediaalta, clasificados en los estadios 3-4 (Beherensmeyer, 1978). Las fracturas halladas con mayor frecuencia fueron aquellas que comprometían la diáfisis de huesos largos, observándose principalmente en sentido trasversal y de forma irregular. En cuanto a la temporalidad todas fueron clasificadas como tafonómicas, producidas en el hueso en estado seco, muchas de las cuales se debieron a la acción de la maquinaria agrícola que posibilitó el descubrimiento del sitio.

Las marcas observadas se condicen en su mayoría con la acción de roedores, dispuestas en las diáfisis de huesos largos en forma concentrada y exhibiendo distintas profundidades. A su vez, uno de los fémures presenta una marca circular (puncture) probablemente generada por la acción de un carnívoro en el sector proximal del hueso. Sólo en cuatro diáfisis de huesos largos se observaron marcas atribuibles a la acción antrópica, todas ellas de corta longitud, con poca profundidad y de disposición oblicua o transversal al eje longitudinal del hueso (Fig. 3). En numerosas piezas óseas se relevó la presencia de carbonato de calcio, lo cual otorga al conjunto óseo una coloración blanquecina impidiendo el relevamiento de ciertos rasgos anatómicos.

El grado de preservación descrito dificultó la caracterización biológica de los individuos, no obstante se lograron establecer parámetros para su concreción. El conjunto óseo está conformado por un total de 1025 elementos óseos $(\mathrm{NISP}=1025)$ (Tabla 1). Se estimó el Número Mínimo de Individuos, el cual fue de 15 individuos (NMI=15) a partir de los huesos temporales izquierdos. De acuerdo a los parámetros ya establecidos se determinó que 13 de ellos eran de edad adulta (mayores de 20-22 años) y 2 subadultos. A partir del grado de formación y erupción dentaria se estimó que uno de los individuos subadultos tendría una edad de muerte de 2 años \pm 8 meses, clasificado como infantil; mientras que el subadulto restante superaría los 12 años de edad (adolescente).

Fue posible realizar el análisis morfológico 


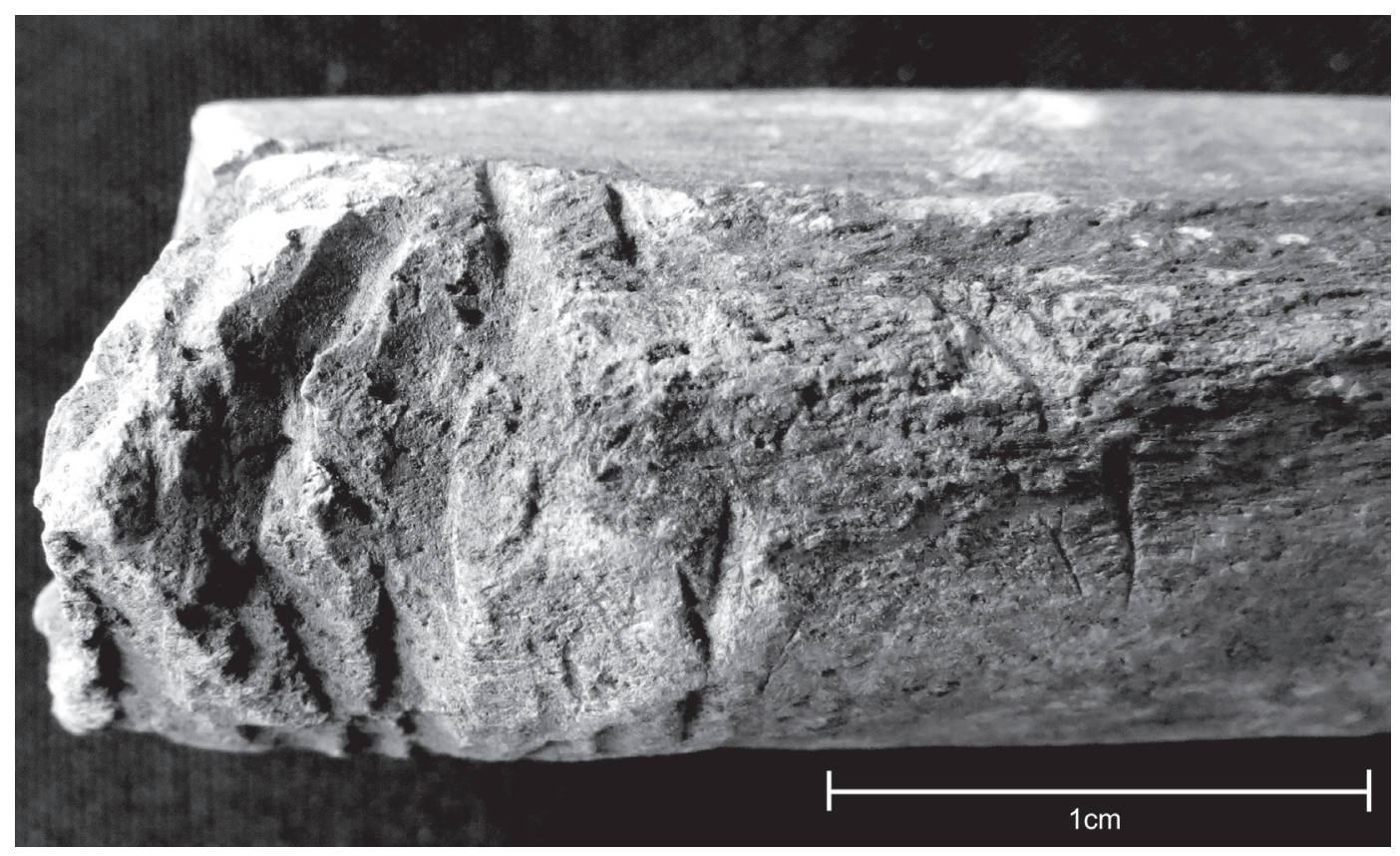

Fig. 3. Marcas de corte en diáfisis femoral.

craneal para determinar el sexo en nueve individuos dando como resultado que cinco individuos adultos pertenecen al sexo masculino, tres al femenino y uno de sexo indeterminado (en los restantes cuatro casos no se pudo efectuar el análisis debido al estado de preservación del material (Fig. 4). Como ya se destacó, esta misma situación imposibilitó la determinación del sexo de los dos individuos subadultos.

Solo fueron halladas señales osteopatológicas en los restos de individuos adultos. Las más frecuentes resultaron aquellas que afectaban la cavidad bucal, agrupadas en enfermedades periodontales e infecciosas (Fig. 5). Las pérdidas de hueso alveolar observadas fueron: de tipo horizontal (o periodontitis) en 26 alvéolos correspondientes a maxilar superior y en 20 pertenecientes a fragmentos mandibulares; y de tipo vertical que comprenden fenestraciones y dehiscencias, registradas en 15 alvéolos del maxilar superior y 14 del maxilar inferior.

Las enfermedades infecciosas relacionadas con el aparato masticatorio se manifestaron en forma de caries y abscesos. Los procesos cariogénicos hallados fueron en tres molares superiores, uno de carácter leve y dos de grado severo afectando la cavidad pulpar; mientras que los abscesos observados fueron cuatro, dos de ellos en relación con las lesiones cariogénicas anteriormente descritas.

Las patologías de tipo osteoarticular observadas se remitieron exclusivamente a la columna vertebral, a excepción de una osteofitosis observada en las carillas articulares para la $1^{\circ}$ costilla de un manubrio esternal. En las vértebras en tanto se observaron dos de ubicación torácica con osteofitosis moderada (Fig. 6) y dos apófisis espinosas anquilosadas, probablemente por efecto de una osteoartrosis severa. En dos fragmentos pertenecientes a vértebras torácicas se observaron lesiones compatibles con hernias discales verticales o nódulos de Schmörl, en ambos casos de grado 1 o leve.

Fueron observados signos de enfermedades infecciosas inespecíficas en ocho elementos óseos. Un fragmento distal de húmero derecho presentó signos probables de osteomielitis, mostrando pequeñas fístulas de drenaje en su área cortical. Se relevaron además cuatro fragmentos de tibia, uno de fémur izquierdo, uno de fémur derecho (Fig. 7) y uno de húmero izquierdo con periostitis marcada. Por último cabe destacar que no se registraron señales atribuibles a traumas ni a patologías metabólicas. 


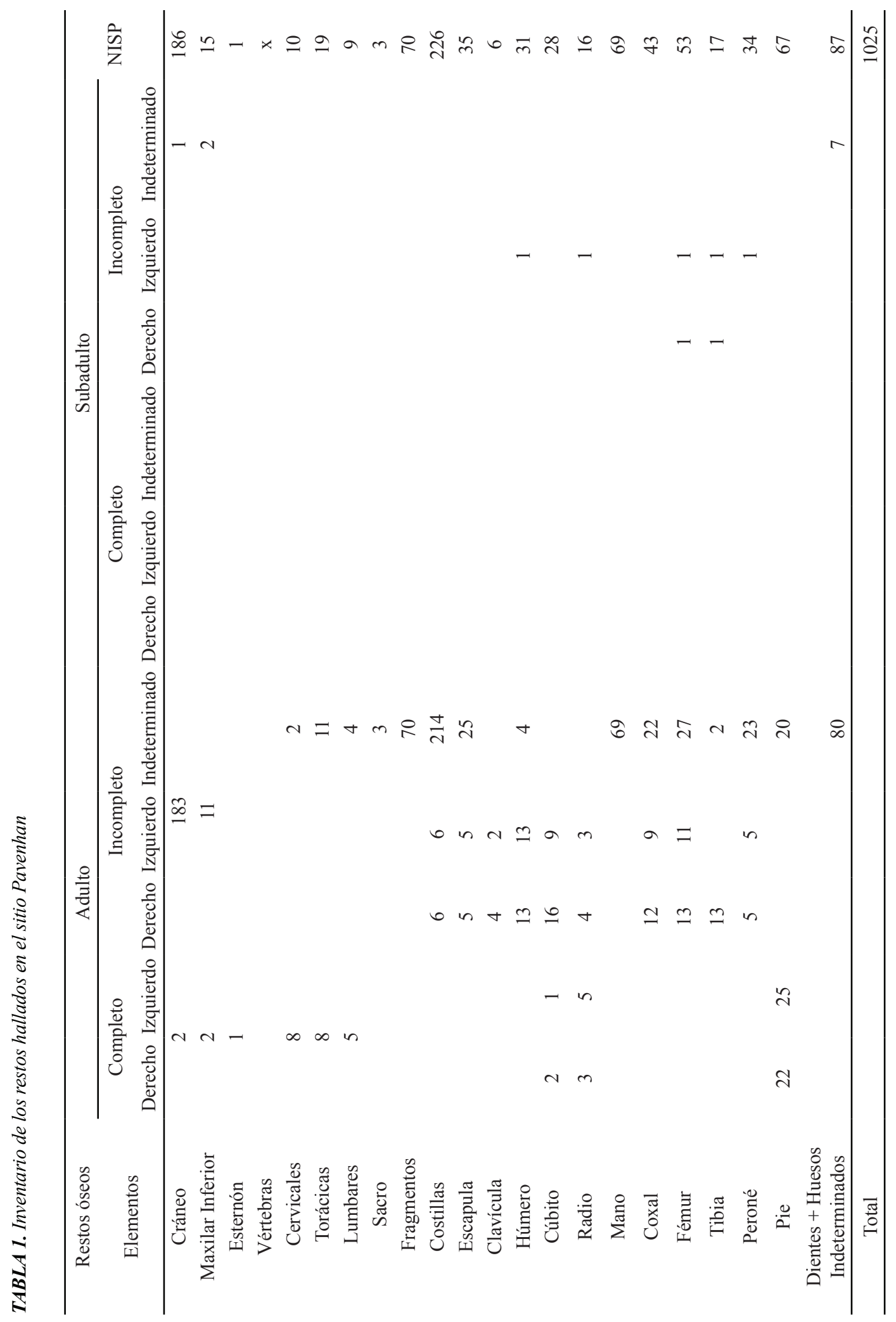




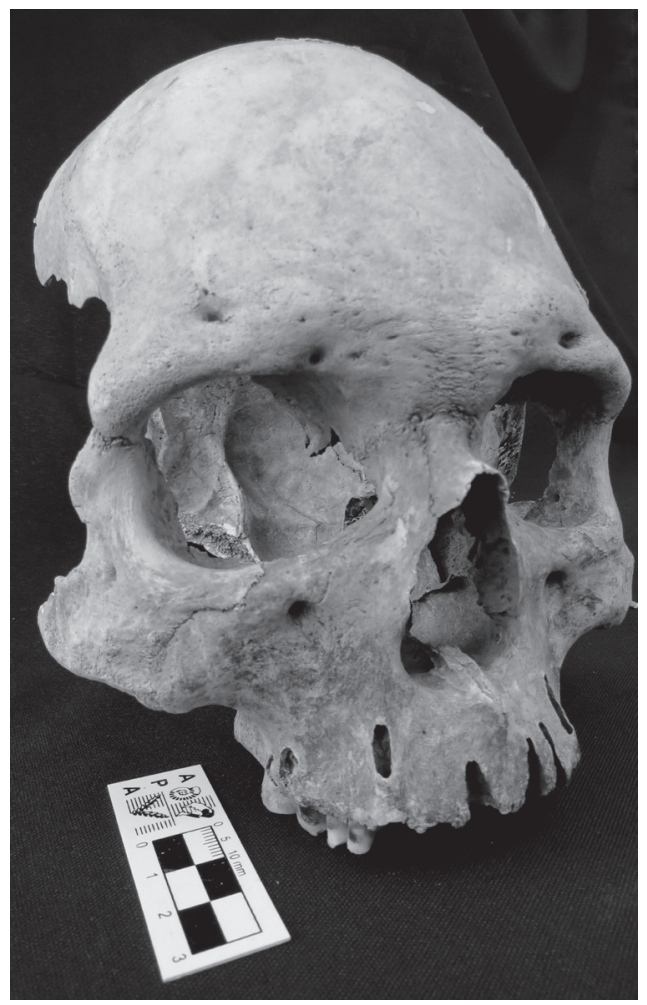

Fig. 4. Cráneo fragmentado perteneciente a un individuo de sexo masculino.

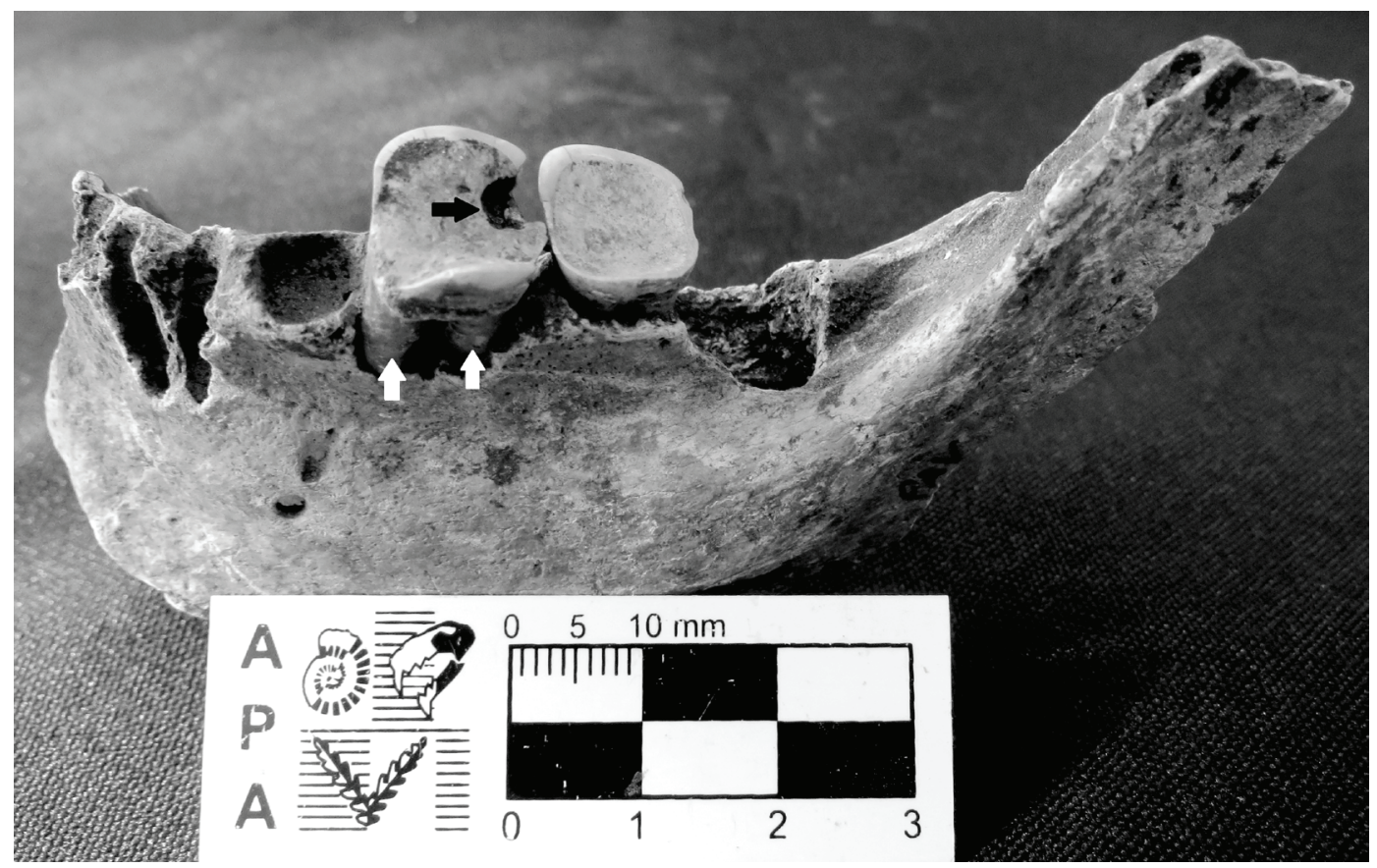

Fig. 5. Patologías afectando piezas dentarias el $1^{\circ}$ molar inferior derecho en maxilar inferior. Caries (flecha negra), Periodontitis (flechas blancas). 


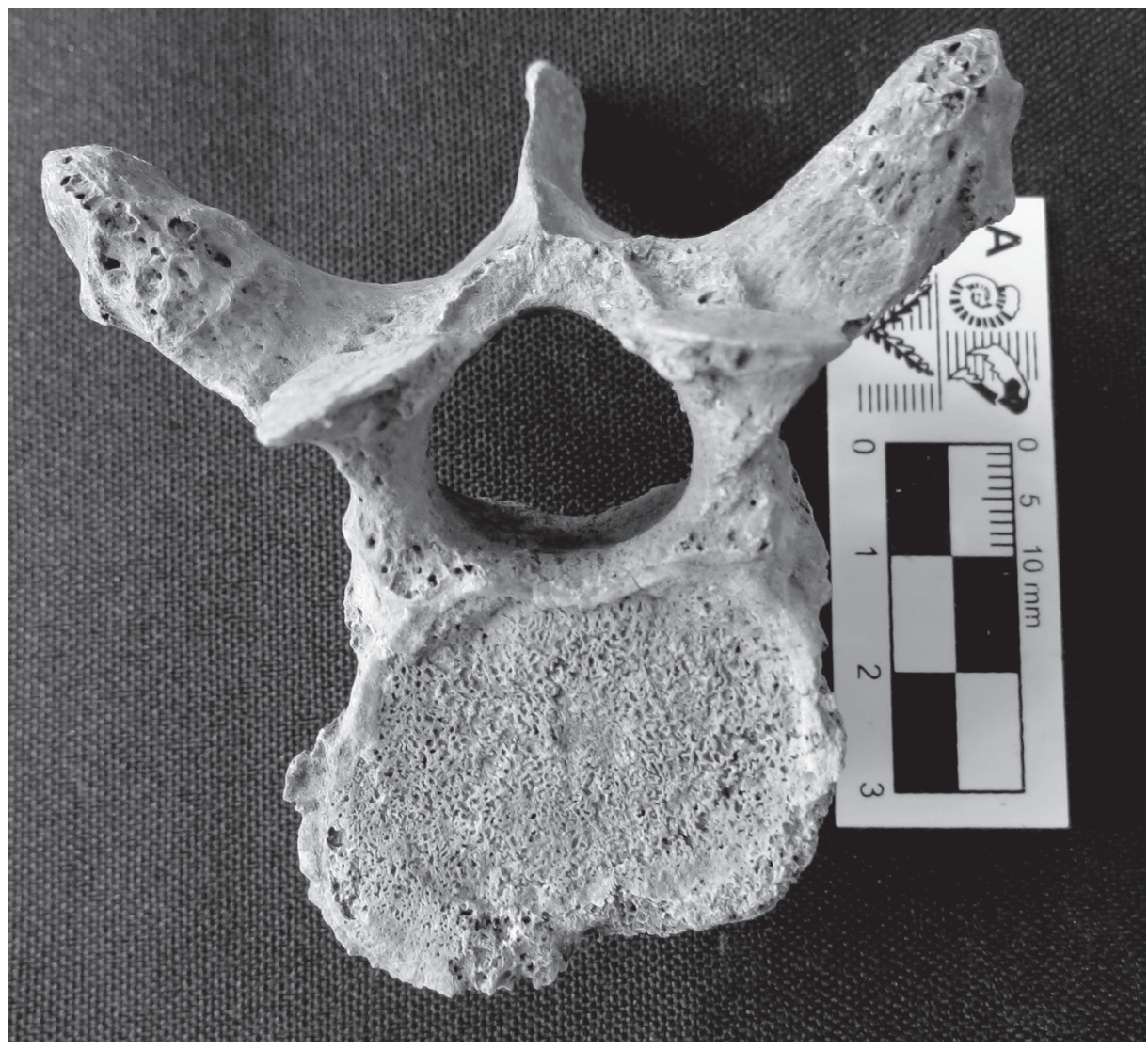

Fig. 6. Osteoartrosis vertebral.

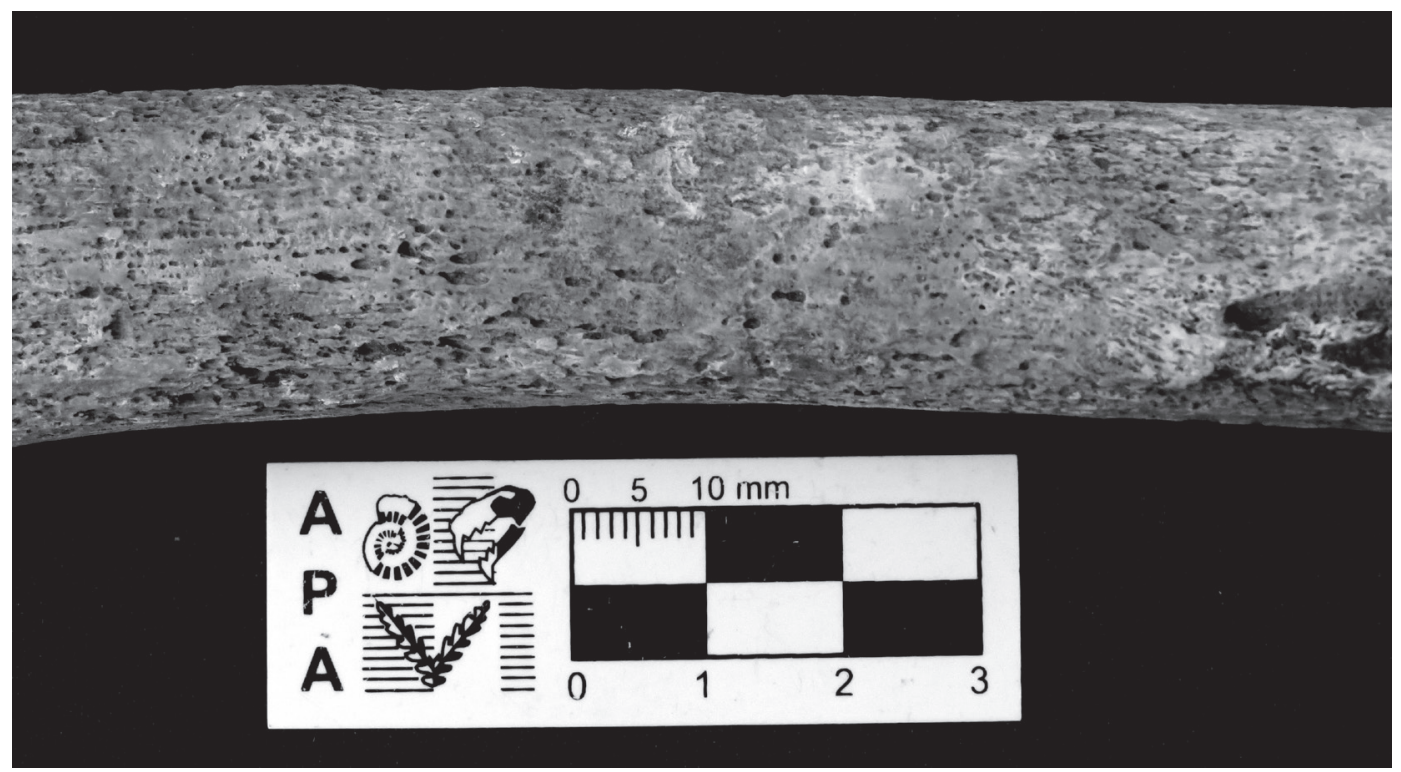

Fig. 7. Periostitis en diáfisis femoral. 


\section{DISCUSIÓN}

Este sitio arqueológico es interpretado como un enterratorio secundario múltiple (sensu Ubelaker, 1999; White et al., 2012). La mayor dificultad afrontada al momento de analizar este tipo de contextos en general y el caso de Pavenhan en particular es el alto grado de fragmentación que presentan los restos óseos. Los huesos desarticulados representan un desafío para el antropólogo, y responden a factores que van desde la disturbación accidental hasta manipulación ritual de los restos (McKinley, 2004). Existen una multiplicidad de medidas de abundancia taxonómica y anatómica que generalmente se utilizan para el análisis cuantitativo en entierros secundarios o de restos óseos mezclados (Flensborg, 2012). Su formulación proviene de la paleontología y zooarqueología (Lyman, 1994; Mengoni Goñalons, 1988), aunque desde hace décadas son utilizados en contextos inhumatorios de poblaciones humanas (Christensen, Passalacqua y Bartelink, 2014; Mays, 1998). El número de especímenes identificados por taxón (NISP=1025) da cuenta sobre la estructura cuantitativa de la muestra (Luna, 2008; Mengoni Goñalons, 1988), a la vez que evidenció la alta fragmentación del registro bioarqueológico. El NMI, por su parte, permitió estimar cuál fue la cantidad mínima de individuos involucrados en el conjunto óseo hallado (Mays 1998, White et al., 2012). Sin embargo, el cálculo del NMI se complejiza cuando los restos fragmentados son mayoría como es el caso aquí descripto (Mays 1998; Salado Puerto, Egaña, Doretti y Vullo, 2014), debiendo considerar en primer término que todos los fragmentos tengan el mismo rasgo anatómico individualizante para asegurarse que no pertenecen al mismo elemento. Si bien el cálculo de NMI es una práctica rutinaria en el análisis de inhumaciones múltiples, la misma no siempre da cuenta fehacientemente de la población original involucrada en el sitio, puesto que no considera los procesos postdepositacionales que allí ocurrieron. En este sentido, en los últimos años comenzó a estimarse cada vez con mayor frecuencia el número más aproximado de individuos (MLNI por el inglés Most Likely Number of Individuals) (Konigsberg y Adams, 2014). Dicho índice se calcula teniendo en cuenta las lateralidades de los huesos largos así como los pares de huesos largos del mismo individuo (v. gr. húmero derecho e izquierdo del mismo esqueleto). Estas condiciones, que permiten una mayor precisión en la estimación de la población de origen, son a su vez un criterio de inclusión que muchos sitios no suelen cumplir debido a su alta fragmentación, sea esta por perturbaciones tafonómicas o por acción antrópica al momento de seleccionar y tratar al esqueleto en el caso de entierros secundarios.

Debido a estas limitaciones algunos autores sostienen que, ante tal fragmentación o un pobre estado de preservación en contextos forenses, cualquier método de cuantificación utilizado puede carecer de sentido, convalidando sólo análisis genéticos para intentar un acercamiento más preciso (Budimlija et al., 2003). En contextos arqueológicos existe un consenso en considerar al NMI como la primera aproximación a la estructura demográfica de la muestra, sin dejar de considerar las variables que pueden afectar su estimación (Mays 1998, Salado Puerto et al., 2014).

Para el caso del sitio Pavenhan debemos hacer algunas consideraciones en tanto práctica inhumatoria. En términos generales, la presencia de entierros secundarios presenta una amplia distribución espacio temporal, desde el Holoceno temprano hasta tiempos históricos, en todo el continente sudamericano (Scabuzzo y Politis, 2010). De manera específica, la práctica secundaria ya ha sido registrada en la región chaqueña con una antigüedad que se remonta a los primeros siglos de la Era cristiana hasta tiempo históricos (Lamenza et al., 2012). En la 1lanura aluvial del Paraná medio, Cornero (2014) y Vaiana y Cornero (2017) en el departamento San Javier, en el sitio arqueológico El Camping presenta numerosos enterratorios primarios y secundarios con diferentes cronologías. Feuillet Terzaghi y Deluca (2011) documentaron entierros secundarios en el sitio Río Salado-Coronda II (Santo Tomé), diferenciando acumulaciones de huesos largos y pocos cráneos y acumulación de hasta tres cráneos, con escasos huesos largos asociados. En el ecotono con el espinal, en el sitio La Lechuza, también se han documentado entierros secundarios de paquetes y huesos aislados (Cornero, 1999), al igual que al sur de la provincia, en el sitio Laguna El Doce (Avila y Ceruti, 2013). En el Delta inferior del Río Pa- 
raná, se han discriminado tres modalidades de entierros secundarios (Mazza y Loponte, 2012), describiendo paquetes funerarios, acumulaciones óseas y cráneos aislados o conjuntos de ellos, mientras que para el Delta superior se han descrito tanto paquetes funerarios como huesos aislados en el sitio Los Tres Cerros I, entre muchos otros (ver Scabuzzo, Ramos van Raap, Bonomo y Politis, 2015). Aun así, en el caso particular de Pavenhan, la disposición especial de los restos con ordenamiento diferencial de huesos largos en relación con los cráneos es completamente novedosa para la región. Solo encontramos un caso que podría compararse en el ámbito del Paraná medio en la margen izquierda del Arroyo Malo, donde se describen inhumaciones secundarias de la Entidad Cancha de Luisa agrupadas en dos paquetes con los huesos largos ordenados en filas paralelas y de manera transversal las costillas con los que se formó una plataforma sobre la que se dispusieron los cráneos (De Brito y Vulcano, 1985). Por lo tanto podemos destacar por un lado la disposición de los restos que marca una identidad particular y por otro, algunas consideraciones generales que son compartidas por las poblaciones humanas que implementaron prácticas secundarias. Para ese comportamiento mortuorio es necesaria la esqueletización y desarticulación del individuo, la selección de los restos, la disposición del lugar de inhumación, entre otras actividades rituales relacionadas (Goldstein, 1981). La conducta mortuoria es vista como altamente dinámica y manipulada por la comunidad en función de su identidad e intereses políticos e ideológicos (Hodder, 1984; Schroeder, 2001). Por otra parte, la incidencia de entierros de este tipo en sociedades cazadoras-recolectoras puede estar en relación con su grado de movilidad (Byrd y Monahan, 1995). Estudios etnográficos señalan que en un gran porcentaje de los casos el momento del entierro secundario funciona como un cierre al período de luto, denotando la complejidad de las sociedades que lo llevan a cabo (Rosenblatt, Walsh y Jackson, 1976). En este sentido cobra especial significación aquella imagen descripta con el Padre Dobrizhoffer sobre el manejo de los restos óseos por parte de los Abipones. En su convivencia con ellos ha presenciado la muerte en combate de un cacique cuyos restos fueron descarnados en campo de batalla, los hue- sos guardados en un saco de cuero y trasladados hasta la comunidad. Las ceremonias fúnebres duraron nueve días hasta que los restos fueron enterrados. Este lugar de inhumación no necesariamente responde a su locación definitiva.

....Suelen agregar a los huesos de sus abuelos los despojos de los compatriotas que ocultaron aquí y allá, en sus perpetuas peregrinaciones. Asi sucede que los desentierran, transportan y recorren inmensas distancias para por fin dejarlos descansar en el atávico y silvestre mausoleo de su raza. Distinguen estos monumentos no por epitafios, ya que desconocían las letras, sino por ciertas notas grabadas en los árboles y otras señales heredadas de sus mayores..." (Dobrizhoffer [1784]1968:298)

Estas ceremonias y prácticas no eran exclusivas de los jefes sino que todos los caídos en batalla eran descarnados y trasladados sus huesos,

....Si ven que alguno de sus compañeros cae durante el combate, rescatan de entre los enemigos su cuerpo exánime para exhumarlo en el suelo patrio de acuerdo a sus ritos. Pero para evitar el peso del cuerpo mientras realizan el camino, descarnan los huesos, enterrando la carne donde pueden..." (Dobrizhoffer [1784]1968:297).

El Padre Florian Paucke describe una situación similar para los Mocoví que él conoció en marco de la reducción de San Javier,

...Después del tiempo en que ellos creen que el cuerpo se pudre y sólo quedan los huesos, viajan al sitio, sacan los huesos y los trasladan al lugar donde están sepultados sus compañeros de estirpe, aunque ellos tuvieren que viajar hasta doscientas y más leguas para ello...cuando los indios parten hacia un distante lugar espanol o a un diferente dominio indio para librar allá un combate y tienen que emprender la huida, dejan echados sobre el campo sus muertos que ellos no pueden arrastrar consigo hasta que ellos creen que los esqueletos yazgan sin carne sobre el campo; luego se ponen en movimiento los amigos de los muertos y viajan por tan largo camino para juntar únicamente los esqueletos de sus compatriotas y transportarlos... (Paucke [1769] 2010:340).

La importancia de los huesos no es exclusiva de la muerte en combate y la práctica de transportarlos hasta el lugar de sepultura no se interrumpió por la evangelización, 
....el tigre saltó contra él con tanta rapidez que él erró el lanzazo y el tigre le aplicó un mordiscón en la nuca por lo cual él se desmayó. Pero después que el [tigre] se había alejado hasta cincuenta pasos de él, comenzó seguir provocando al tigre que de nuevo le ha asaltado y lo ha muerto con el segundo mordiscón. Sus escasos compañeros mondaron la carne de sus huesos, la enterraron y trajeron los huesos mondados al pueblo a enterrar en el campo de Dios como se suele decir..." (Paucke [1769] 2010:341).

Para finalizar, debemos destacar la importancia de este tipo de hallazgos para aproximar al conocimiento sobre los modos de vida de las poblaciones humanas. Aunque la elevada fragmentación y dispersión de los esqueletos no permitió su correlación con variables biológicas como la edad y el sexo de los individuos, los rasgos patológicos relevados cobran relevancia en relación a la alimentación de los grupos del área. En cuanto a la salud bucal, la marcada presencia de signos de enfermedad periodontal, si bien inespecífica, coincide con lo observado en sitios arqueológicos cercanos a cuerpos de agua tanto marítimos como fluviales (Lamenza y Plischuk, 2015; Machado y Kneip, 1994). La presencia de lesiones severas con un origen cariogénico y los abscesos encontrados indicarían un consumo moderado de hidratos de carbono (Drube, 2008; Ortner, 2003). Sin embargo, la ausencia de señales de patologías metabólicas como criba orbitalia, hiperostosis porótica o hipoplasia dental, permiten conjeturar un modo de subsistencia carente de agricultura intensiva. La baja prevalencia de estas patologías es una constante en la región; esqueletos vinculados con la Entidad Arqueológica Goya - Malabrigo (18101480AP) (Del Papa, Scabuzzo, Ramos van Raap, Bonilla y Pennini, 2016), sitios del sur de la región del NEA durante el Holoceno tardío (Ramos van Raap, 2018; Scabuzzo et al., 2015); Laguna el Doce en el sur de la provincia de Santa Fe (Piccoli, 2009) y sitios ubicados en la 1lanura aluvial del Paraná (2000-1000AP) (Cornero y Puche, 2000) evidencian, al igual que en Pavenhan, una escasa prevalencia de indicadores de estrés metabólico prolongado y una moderada aparición de caries.

Esta hipótesis es apoyada por la investigación de Bollini, Atencio, Méndez y Lamenza
(2014) al analizar específicamente el desgaste de las piezas dentales de los individuos del sitio Pavenhan. En dicho trabajo los autores observan una angulación en las caras oclusales, patrón que atribuyen a un tipo procesamiento de alimentos mediante molienda, a la vez que describen la presencia de cupping, indicador del aporte de partículas abrasivas en el proceso de preparación o consumo. Estas partículas podrían provenir tanto del uso de morteros como de granos de arena adosados a recursos acuáticos (Kieser et al., 2001). En dicha oportunidad se resaltaron las fracturas dentales observadas, relacionándolas con sociedades agrícolas y en dientes con presencia de cupping. Aún así cabe considerar la posibilidad de una intensificación en el procesamiento de productos recolectados sin necesidad de estar relacionados con la producción de alimentos. En este sentido, el patrón de desgaste identificado se diferenciaría de lo descrito para sitios más tempranos al sur de la provincia (Piccoli, 2009), con un desgaste más semejante al tipo cazador-recolector, caracterizado por presentar una atrición más pronunciada, con formas acentuadas y planos, principalmente en la dentición anterior (Bollini et al., 2014; Deter, 2009; Kieser et al., 2001; Smith, 1984). Por su parte, los sitios del sur del NEA analizados por Ramos van Raap (2018), muestran individuos con patrones de desgaste mixtos, con características propias de cazadores-recolectores como también de consumo de alimentos procesados por molienda.

Las patologías osteoarticulares halladas, osteoartrosis y nódulos de Schmörl, no presentaron rasgos particulares en su prevalencia, distribución y severidad, por lo cual no fue posible establecer su relación con algún tipo de actividad física, aunque cabe aclarar que la ausencia de rangos de edad más acotados dificultó dicho análisis. Este hallazgo es similar a lo relevado por Scabuzzo y colaboradores (2015) para la localidad arqueológica de Tres Cerros (1227560AP). Si bien allí la prevalencia es mayor $(27 \%)$, tampoco se evidencia una relación directa con un tipo particular de actividad. En sitios prehispánicos de Santa Fe la situación es similar, Cornero (1999) también encuentra escasos signos artrósicos para La Lechuza, situado en el norte de la provincia (1760AP).

Por último, es remarcable la aparición lesio- 
nes atribuibles a patologías infecciosas inespecíficas. Similares hallazgos fueron relevados para Tres Cerros (Scabuzzo et al., 2015), sin que hasta el momento se haya establecido con certeza los procesos causantes de dicha patología, aunque en el mismo sitio Ramos van Raap (2018) reporta un caso probable de treponematosis. El incremento en signos de enfermedades infecciosas ha sido vinculado con el cambio en los modos de vida por diversos autores. Rodríguez Cuenca (2006) describe cuatro causas sociales que generarían este aumento en las prevalencias: aumento de densidad y concentración poblacional, el hacinamiento e higiene deficiente, insuficiencia de alimentos, y el contacto estrecho con agentes transmisores de patógenos. Cockburn (1964) fue una de las primeras en postular la aparición de esta clase de patologías en relación con la domesticación de animales durante el neolítico. Los grupos cazadores-recolectores nómades, al conformarse como comunidades mayores en un pequeño territorio, en condiciones cada vez menos higiénicas y con menor disponibilidad de agua, habrían sufrido la emergencia de múltiples patologías infecciosas (Waldron, 2009). Incluso el principal agente infeccioso en estos grupos son parásitos intestinales, aunque estos no afectan el material esqueletario y su detección arqueológica depende de otros métodos (Ortner, 2003). Desde la perspectiva del agente infeccioso es más sencilla la supervivencia y reproducción en grandes grupos sedentarios, con alta densidad poblacional como las sociedades agrícolas, que en un grupo de pocos individuos con alta movilidad como los cazadores recolectores (Katzemberg, 2012). Si bien en Pavenhan no se han encontrado en el registro indicios de agricultura, las características de su inhumación y el número de individuos hallado podrían indicar un aumento en la densidad poblacional que habría generado condiciones sanitarias deficientes, menor cantidad de agua disponible, déficit nutricionales, y un mayor hacinamiento, condiciones que generan un incremento en la prevalencia de patologías infecciosas (Cohen y Crane-Kramer, 2003; Roberts y Manchester, 1995; Suby, 2012). Cabe mencionar que en ninguna de las fuentes documentales mencionadas anteriormente se desprende que esta forma de vida haya sido característica de los pueblos de esta región en tiempos prehispánicos.

\section{CONCLUSIONES}

El análisis del material osteológico recuperado indica que en Pavenhan fueron inhumados de manera secundaria al menos 15 individuos entre adultos e infantes, sin selección diferencial de edades o sexos, con una disposición novedosa para la región. En el orden metodológico queda nuevamente demostrada la valía de las tareas de conservación in situ y de las actividades de limpieza y remontaje, las cuales permitieron el estudio del material en el laboratorio (Martínez, Flensborg y Bayala, 2009). En particular toda técnica que conduzca a mejorar la precisión de la estimación del NMI es vital, al ser un ítem crítico en estudios paleodemográficos para la interpretación de culturas pasadas (Konigsberg y Adams, 2014).

La información contextual y los resultados del análisis osteopatológico refuerzan la propuesta de que para momentos previos a la conquista española, las sociedades cazadoras recolectoras pescadoras que habitaban el Chaco austral practicaban de manera generalizada enterratorios secundarios con particularidades que podrían deberse a diferencias culturales y contaban con una incorporación incipiente de hidratos de carbono. En cuanto a las características de la dieta de estas poblaciones, estudios reportaron el consumo de plantas domesticadas para el noreste santafesino hacia el 1700-2000 AP (Cornero y Rangone, 2015), a partir del hallazgo de fitolitos de gramíneas y almidones extraídos de cálculo dentario, mientras que en sitios de la costa oriental del Paraná el registro de este consumo está ampliamente documentado para los últimos 1000 años (ver Ramos van Raap, 2018), antecedentes que también alertan sobre la posibilidad del mismo tipo de consumo para los individuos vinculados con el sitio Pavenhan.

\section{AGRADECIMIENTOS}

Al Prof. Dante Ruggeroni. Al Dr. Mariano Santini por realizar el análisis de preservación del material.

\section{LITERATURA CITADA}

Avila, J. D. y Ceruti, C. N. (2013). El Holoceno Tempra- 
no-Medio y la ruta del poblamiento: Laguna el Doce, Departamento General López, Provincia de Santa Fe, Argentina. Cuadernos del Instituto Nacional de Antropología y Pensamiento Latinoamericano - Series Especiales, 4, 21-33.

Behrensmeyer, A. K. (1978). Taphonomic and ecological information from bone weathering. Paleobiology (4), 150-162. doi:10.1017/S0094837300005820

Bollini, G., Atencio, J. P., Méndez, M. G y Lamenza, G. (2014). Primeras aproximaciones al análisis descriptivo del desgaste dental en restos bioarqueológicos del sitio Pavenhan, Chaco Santafesino. Revista Facultad de Ciencias Exactas, Físicas y Naturales, 1, 87-96.

Budimlija, Z. M., Prinz, M. K., Zelson-Mundorff, A., Wiersema, J., Bartelink, E., MacKinnon, G.,...Shaler. R. C. (2003). World Trade Center human identification project: experiences with individual body identification cases. Croatian Medical Journal, 44, 259-263.

Buikstra, J., y Ubelaker, D. (1994). Standards for data collection from human skeletal remains. Fayetteville, Estados Unidos: Archaeological Survey Research Series $N^{\circ} 44$.

Byrd, B. F. y Monahan, C. M. (1995). Death, mortuary ritual, and Natufian social structure. Journal of Anthropological Archaeology, 14, 251-287. doi:10.1006/ jaar.1995.1014

Campillo, D. (2001). Introducción a la Paleopatología. Barcelona, España: Edición Bellaterra S.L.

Cardoso, H. F. V., Vandergugten, J. M. y Humphrey, L. T. (2017). Age estimation of immature human skeletal remains from the metaphyseal and epiphyseal widths of the long bones in the post-natal period. American Journal of Physical Anthropology, 162, 19-35. doi:10.1002/ ajpa.23081

Christensen, A., Passalacqua, N. y Bartelink, E. (2014). Forensic anthropology: current methods and practice. Oxford, Reino Unido: Academic Press. doi:10.1016/ B978-0-12-418671-2.00005-7

Cockburn, T. A. (1964). The evolution and erradication of infectious diseases. Perspectives in Biology and Medicine, 7, 498-499. doi:10.1353/pbm.1964.0020

Cohen, M. y Crane-Kramer, G. (2003). The state and future of paleoepidemiology. En Greenblatt, C. L. y Spigelman M. (Eds). Emerging pathogens: the archaeology, ecology, and evolution of infectious disease (pp. 67-69). New York, Estados Unidos: Oxford University Press.

Cornero, S. (1999). Enterratorios humanos en el litoral: Sitio La Lechuza, Provincia de Santa Fe. Actas del XII Congreso Nacional de Arqueología (pp. 384-388). La Plata, Argentina: Editorial de la Universidad Nacional de La Plata.

Cornero, S. (2014). El camping: rescate y cronologías para un sitio Goya-Malabrigo tardío. Alejandra, Dpto. San Javier, Provincia de Santa Fe. Divulgación de la Producción Científica y Tecnológica de la UNR, VI, 507-511.

Cornero, S. y Puche, R. (2000). Diet and nutrition of prehistoric populations at the alluvial banks of the Paraná River. Medicina, 60, 109-114.

Cornero, S. y Rangone, L. (2015). Análisis arqueobotánicos en sitios de la entidad arqueológica Goya-Malabrgio ubicados en el centro-norte de Santa Fe. Anuario de Arqueología, 7, 85-94.

De Brito, A. y Vulcano, C. (1985). Arqueología de un Yacimiento Indígena. Revista del Centro de Estudios Hispanoamericanos AMERICA, 4, 93-104.

Del Papa, M., Scabuzzo, C., Ramos van Raap, M. A., Bonilla, D. y Pennini. V. (2016). Nuevos análisis bioar- queológicos de la colección osteológica del sitio Paraná Ibicuy 1 (Delta Inferior del Río Paraná, Entre Ríos). Intersecciones en Antropología, 17, 263-267.

Deter, C. (2009). Gradients of occlusal wear in hunter-gatherers and agriculturalists. American Journal of Physical Anthropology, 138, 247-254. doi: 10.1002/ajpa.20922

Dobrizhoffer, M. [1784] (1968). Historia de los Abipones II. Resistencia, Argentina: Facultad de Humanidades, Universidad Nacional del Noroeste.

Drube, H. (2008). Las poblaciones aborigenes prehispánicas de Santiago del Estero. Evaluación de sus características bioantropológicas y de sus condiciones de salud, enfermedad y nutrición (Tesis de Doctorado). Facultad de Ciencias Naturales y Museo, Universidad Nacional de La Plata, La Plata.

Feuillet Terzaghi, M. R. y Deluca, S. L. (2011). Arqueología en la cuenca inferior del río Salado (Pcia. de Santa Fe). Primeras aproximaciones al estudio de un sitio arqueológico con enterratorios múltiples. Folia Histórica del Nordeste, 19, 23-50.

Flensborg, G. (2012). Análisis Paleopatológico en el curso inferior del río Colorado (Pcia. De Buenos Aires). Exploración y evaluación del estado de salud de sociedades cazadoras recolectoras en el Holoceno tardio (Tesis de Doctorado). Facultad de Ciencias Sociales, Universidad Nacional del Centro de la Provincia de Buenos Aires, Olavarría.

Galera, V., Ubelaker, D. y Hayek, L. (1998). Comparison of macroscopic cranial methods of age estimation applied to skeletons from the Terry Collection. Journal of Forensic Sciences, 43, 933-939. doi:10.1520/JFS14337J

Goldstein, L. G. (1981). One-dimensional archaeology and multi-dimensional people: Spatial organization and mortuary analysis. En Chapman, R. y Randsborg, K. (Eds.), The archaeology of death (pp. 53-69). Cambridge, Reino Unido: Cambridge University Press.

Hillson, S. W. (2000). Dental pathology. En Katzenberg, M. A. y Saunders S. R. (Eds.), Biological anthropology of the human skeleton (pp. 249-286). New York, Estados Unidos: Wiley-Liss. doi:10.2458/azu js rc.55.16783

Hodder, I. (1984). Burials, houses, women and men in the European Neolithic. En Miller, D. y Tilley, C. (Eds.). Ideology, Power and Prehistory (pp. 51-68). Cambridge, Reino Unido. Cambridge University Press. 10.1017/ CBO9780511897443.006

Hogg, A., Hua, Q., Blackwell, P., Buck, C., Guilderson, T., Heaton, T.,...Zimmerman, S. (2013). "SHCal13. Southern Hemisphere Calibration, 0-50,000 years cal BP”. Radiocarbon, 55, 1889-1903. doi:10.2458/azu_ js_rc.55.16783

Hoppa, R. D. y Gruspier, K. L. (1996). Estimating diaphyseal length from fragmentary subadult skeletal remains: Implications for paleodemographic reconstructions of a southern ontario ossuary. American Journal of Physical Anthropology, 100, 341-354. doi:10.1002/(SICI)10968644(199607)100:3<341::AID-AJPA3>3.0.CO;2-X

Jiménez-Brobeil, S., Souich, P. y Al Oumaoui, I. (2009). Possible relationship of cranial traumatic injuries with violence in the south-east Iberian Peninsula from the Neolithic to the Bronze Age. American Journal of Physical Anthropology, 140, 465- 475. doi:10.1002/ ajpa. 21089.

Katzemberg, M. A. (2012). The ecological approach: understanding past diet and the relationship between diet and disease. En Grauer, A. (Ed). A companion to paleopathology (pp. 97-113). Chichester, Reino Unido: Blackwell Publising Ltd. doi:10.1002/9781444345940. ch6 
Kieser, J., Dennison, J., Kaidonis, J., Huang, D., Herbison, P. y Tayles, N. (2001). Patterns of dental wear in the early Maori dentition. International Journal of Osteoarchaeology, 11, 206-217. doi:10.1002/oa.534

Konigsberg, L. y Adams, B. (2014). Estimating the number of individuals represented by commingled human remains: a critical evaluation of methods. En Adams, B. y Byrd, J. (Eds.). Commingled human remains (pp. 193-220). Oxford, Reino Unido: Academic Press. doi:10.1016/B978-0-12-405889-7.00009-5

Lamenza, G. y Plischuk, M. (2015). Avances en bioarqueología del Chaco boreal. Arqueología Iberoamericana, $28,75-80$.

Lamenza, G., Rodríguez Mir, J., Calandra, H. y Salceda, S. (2012). Espacio funerario en el Chaco Meridional. En E. Silvera de Buenader y S. Martínez de Montiel (Eds.). El Hombre, el medio y sus relaciones (pp. 177 189). San Fernando del Valle de Catamarca, Argentina: Universidad Nacional de Catamarca.

Lloveras, L., Rissech, C. y Rosado, N. (2016). Tafonomía forense. En Sanabria-Medina, C. (Ed.). Patología y antropología forense de la muerte: la investigación cientifico-judicial de la muerte y la tortura, desde las fosas clandestinas, hasta la audiencia pública (pp. 453-523). Bogotá D.C., Colombia: Forensic Publisher.

Lovell, N. (1997). Trauma analysis in paleopathology. Yearbook of Physical Anthropology, 40, 139-170. doi:10.1002/(SICI)1096-8644(1997)25+<139::AIDAJPA6 $>3.0$. CO $; 2-\% 23$

Luna, L. (2008). Estructura demográfica y relaciones biológicas de cazadores recolectores en un ambiente de desierto. Sitio Chenque I (Parque Nacional Lihué Calel, Pcia. de La Pampa, Argentina). Oxford, Reino Unido: BAR Internacional Series.

Lyman, R. (1994). Vertebrate taphonomy. Cambridge Manuals in Archaeology. Cambridge, Reino Unido: Cambridge University Press. doi:10.1017/ CBO9781139878302

Machado, L. y Kneip, L. (1994). Padrões dentários, dieta e subsistências das populações dos sambaquis de Saquarema, RJ. Revista de Arqueología, 8, 45-57. doi:10.24885/sab.v8i1.463

Martínez, G., Flensborg, G. y Bayala, P. (2009). Estrategias de recuperación y conservación de entierros humanos en el sitio Paso Alsina 1 (curso inferior del río Colorado, Prov. de Buenos Aires, Argentina). Revista Argentina de Antropología Biológica, 11, 95-107.

Mays, S. (1998). The archaeology of human bones. Londres, Reino Unido: Routledge.

Mazza, B. y Loponte, D. (2012). Las prácticas mortuorias en el humedal del Paraná inferior. Arqueología Iberoamericana, 13, 3-21. doi:10.5281/zenodo. 1310875.

McKinley, J. I. (2004). Compiling a skeletal inventory: disarticulated and co-mingled remains. En M. Brickley. (Ed.) Guidance for standards for the recording of human remains (pp. 14-17). Southampton, Reino Unido: British Association for Biological Anthropology and Osteoarchaeology and the Institute of Field Archaeo$\log$.

Mengoni Goñalons, G. (1988). Análisis de Materiales Faunísticos de Sitios Arqueológicos. Xama, 1, 71-120.

Moorrees, C., Fanning, E. y Hunt, E. (1963). Formation and resorption of three deciduous teeth in chidren. American Journal of Physical Anthropology, 21, 205-213. doi:10.1002/ajpa.1330210212

Ortner, D. (2003). Identification of pathological conditions in human skeletal remains. Nueva York, Estados Unidos: Academic Press.
Paucke, F. [1769] (2010). Hacia allá y para acá (memorias). Santa Fe, Argentina: Ministerio de Innovación y Cultura de la Provincia de Santa Fe.

Piccoli, C. (2009). Estudio bioarqueológico de la colección osteológica proveniente del sitio Laguna El Doce (Departamento General López, provincia de Santa Fe). (Tesis de Licenciatura). Facultad de Humanidades y Artes, Universidad Nacional de Rosario, Rosario.

Ramos van Raap, M. A. (2018). Paleopatología en poblaciones prehispánicas del sur del Noreste Argentino. (Tesis de Licenciatura), Facultad de Ciencias Naturales y Museo, Universidad Nacional de La Plata, La Plata.

Roberts, C. y Manchester, K. (1995). The archaeology of disease. New York, Estados Unidos: Cornell University Press.

Rodríguez Cuenca, J. V. (2006). Las enfermedades en las condiciones de vida prehispánicas de Colombia. Maguaré, 20, 265-270.

Rosenblatt, P., Walsh, R. y Jackson, D. (1976). Grief and mourning in cross-cultural perspective. New Haven, Estados Unidos: HRAF Press.

Salado Puerto, M., Egaña, S., Doretti, M. y Vullo, C. (2014). A multidsciplnary approach to commingled remains analysis: anthropology, genetics and background information. En Adams, B.y Byrd, J. (Eds.) Commingled human remains (pp. 7-32). Oxford, Reino Unido: Academic Press: doi:10.1016/B978-0-12-405889-7.00002-2

Scabuzzo, C. y Politis, G. (2010). Entierros secundarios del Holoceno temprano y medio de la región pampeana. Nuevos datos del sitio Arroyo Seco 2. Cazadores- recolectores del cono sur. Revista de Arqueología, 4, 135 155.

Scabuzzo, C., Ramos van Raap, M. A., Bonomo, M., y Politis, G. (2015). Estudios bioarqueológicos en el sitio Los Tres Cerros 1 (Delta Superior del río Paraná, Entre Ríos, Argentina). Boletim do Museu Paraense Emílio Goeldi. Ciências Humanas, 10, 509-535.

Scheuer, L. y Black, S. (2000). Developmental juvenile osteology. London, Reino Unido: Academic Press. doi:10.1016/B978-012624000-9/50004-6

Schroeder, S. (2001). Secondary disposal of the dead: crosscultural codes. World Cultures, 12, 77-93.

Smith B. H. (1984). Patterns of molar wear in huntergatherers and agriculturalist. American Journal of Physical Anthropology, 69, 39-56. doi:10.1002/ ajpa.1330630107.

Stuart-Macadam, P. (1989). Porotic hyperostosis: relationship between orbital and vault lesions. American Journal of Physical Anthropology, 80, 187-193. doi:10.1002/ ajpa.1330800206

Suby, J. (2012). La salud de nuestros antepasados: una mirada sobre la paleopatología. Quequén, Argentina: Laboratorio de Ecología Evolutiva Humana.

Tuller, H. y Hofmeister, U. (2014). Spatial analysis of mass grave mapping data to assist the reassociation of disarticuled and commingled human remains. En Adams, B.y Byrd, J. (Eds.) Commingled human remains (pp. 7-32). Oxford, Reino Unido: Academic Press: doi:10.1016/ B978-0-12-405889-7.00002-2

Ubelaker, D. (1999). Human skeletal remains: Excavation, Analysis, Interpretation. Washington, Estados Unidos: Taraxacum.

Üstündağ, H. (2009). Schmorl's nodes in a post-medieval skeletal sample from Klostermarienberg, Austria. International Journals of Osteoarchaeology, 19, 695-710. doi:10.1002/oa.993

Vaiana, G. y Cornero, S. (2017). Sitio arqueológico El Cam- 
ping, Alejandra, Santa Fe. Actualizaciones. Actas de las XI Jornadas de Ciencia y Tecnología. Divulgación de la Producción Científica y Tecnológica de la UNR (pp. 1591-1598). Rosario, Argentina: UNR Editora.

Waldron, T. (2009). Palaeopathology. New York, Estados Unidos: Cambridge University Press. doi:10.1017/ CBO9780511812569

Weiss, E. (2006). Osteoarthritis and body mass. Journal of
Archaeological Science, 33, 690-695. doi:10.1016/j. jas.2005.10.003

White, T.E. (1953). A method of calculating the dietary percentage of various food animals utilized by Aboriginal peoples. American Antiquity, 18, 396-398. doi: $10.2307 / 277116$

White, T., Black, M. y Folkens, P. (2012). Human osteology. Londres, Reino Unido: Academic Press. 